\title{
Parent-offspring regression and extreme environments
}

\author{
PATRICK J. WARD \\ Unité INSERM de Recherches en Epidemiologie des Cancers, U351, Institut Gustave Roussy, 39 Rue Camille \\ Desmoulins, 94805 Villejuif Cedex, France
}

\begin{abstract}
The parent-offspring regression coefficient (narrow heritability) has often been reported to increase with the extremity of environmental conditions. This is frequently interpreted as evidence for the expression of 'new genes' under novel environmental conditions. Strictly speaking, however, the parameters of the additive-dominance model of quantitative inheritance only have meaning under a given set of environmental conditions. It is not clear what a change of parameter values, in response to a change of experimental conditions, means in terms of biological processes going on beneath the phenotypic level. The parent-offspring regression coefficient is therefore reconsidered here from the perspective of a biological model of gene expression. It is demonstrated that the parent-offspring regression coefficient can increase directly with the average value of the environmental contribution to the phenotype. More generally, an increase in the strength of parent-offspring regression with extremity of the environment does not necessarily indicate that any change has taken place in the genetic basis of phenotypic value.
\end{abstract}

Keywords: extreme environment, heritability, metabolic flux.

\section{Introduction}

There have been many reports of narrow heritability estimates increasing with the 'extremity' of the environmental conditions, i.e. of narrow heritability being relatively greater when the environmental conditions under which the phenotype is expressed are more stressful for the average member of the species. This finding has been reported across a very wide variety of species, traits and environmental factors.

In one of the earliest reports, Crow (1957) found that the population response to selection for insecticide resistance in Drosophila melanogaster accelerated with increasing insecticide dose. Studies of other traits in $D$. melanogaster and of other environmental factors also revealed increases of narrow heritability in response to an increase in the stressfullness of the environment, such as for longevity in response to gamma-radiation dose (Westerman \& Parsons, 1973), for adult survival time in response to ethanol vapour concentration (Parsons, 1982; van Herrewege \& David, 1984) and to acetic acid concentration (Parsons, 1982), for sternopleural cheatae number in response to temperature (Schnee \& Thompson, 1984) and for larval development time in response to citric acid concentration of the larval growth medium (Ward, 1985). Analogous findings have been reported using other organisms and include increased heritability of lead tolerance in the plant Festuca ovina with increasing lead contamination of the soil (Urquhart, 1971), an accelerated response to selection for timing of the first clutch in the cotton stainer bug Dysdercus bimaculatus with increasing moisture stress (Derr, 1980), an increase of narrow heritability for both female development time and for instantaneous mortality rate in the rice weevil Sitophilus oryzae after transfer to a more stressful diet (Holloway et al., 1990) and an increase of narrow heritability for clutch size in the water flea Daphnia magna in response to food deprivation (Ebert et al., 1993).

These findings are consistent with a wider literature reporting increases in the relative strength of the genetic component of the phenotypic variance in response to increased environmental stress, such as for weight in the plant Arabidopsis thaliana in response to increasing temperature stress (Lagridge \& Griffing, 1959), for reproductive performance in mice in response to increasing stress in early pregnancy (Belyaev \& Borodin, 1982) and for human psychomotor performance tasks in response to increasing alcohol dose (Martin et al., 1985). Further references 
can be found in Pani \& Lasley (1972), Barton \& Turelli (1989) and Hoffmann \& Parsons (1991).

These reports are surprising when it is recalled that an extreme environment is, by definition, one to which the species or study population is poorly adapted in a Darwinian sense. Narrow heritability is measured either as the linear regression coefficient relating the average of a parental pair to the average of their offspring (the 'parent-offspring regression coefficient') or equivalently as the population rate of response to selection divided by the strength of the applied selection. It is generally interpreted as the component of the phenotypic variance attributable to variation in additive genetic factors (Bulmer, 1985; Falconer, 1989). Additive genetic factors are considered in turn to represent a part of the phenotypic variance coming directly from variation in the genes (Fisher, 1918). An increase of narrow heritability with the extremity of the environmental conditions would therefore suggest that polymorphic gene loci exist which are only expressed, or which are expressed more strongly, under conditions to which the species is poorly adapted. The data cited above have often been interpreted as such, i.e. as evidence for the expression of 'new genes' under novel environmental conditions. This is quite a strong hypothesis, demanding consideration of how and why such genetic information would evolve. An alternative explanation could be a decrease in the size of the environmental contribution to the phenotypic variance with the extremity of the environment. This explanation is unsatisfactory as it entails the paradox that the ontological development of an individual is less susceptible to environmental perturbation (i.e. is under tighter genetic control) under conditions to which the species is poorly adapted.

Before proposing such biological explanations for these data, however, some clarification is needed concerning the appropriateness of classical quantitative genetics methodologies as a means of inferring changes of gene expression with a change of experimental conditions. Strictly speaking, the empirical values obtained for quantitative genetic parameters are only of interest in the environment and population in which they are made. This is how they are defined (Falconer, 1989). It is not clear what a comparison of quantitative genetic parameters obtained under different sets of environmental conditions tells us about biological processes going on beneath the phenotypic level. The methodology is not based on any biological model of development or gene expression. Parameters such as the breeding value (additive genetic component) of an individual are defined entirely in statistical terms and entirely at the phenotypic level. The additive-dominance model is a pragmatic aid to the description and statistical analysis of quantitative genetic data and its usefulness as a guide to selective breeding is unquestioned but it may nevertheless be a mistake to view the additive-dominance model as a biological model (Ward, 1985, 1990).

The parent-offspring regression coefficient is reconsidered here from the perspective of a biological model of gene expression. This model represents the genetic contribution to the kinetics of biochemical metabolism occurring within an individual. On the basis of this model, it is demonstrated that the parent-offspring regression coefficient can increase directly with the average value of the environmental contribution to the phenotype. From a biological standpoint, this is a more plausible explanation for a relative increase of parent-offspring regression with the extremity of the environmental conditions than is the hypothesis of 'new genes' being expressed under extreme environments. More generally, the present analysis shows that the parent-offspring regression coefficient can increase in strength without any change in the genetic basis of the trait.

\section{Metabolism and quantitative inheritance}

A phenotypic value measured on an individual can be regarded generally as some function of the rate of flow (or 'metabolic flux') through a biochemical network underlying the expression of the trait within that individual (Kacser \& Burns, 1981; Keightley \& Kacser, 1987; Beaumont, 1988; Keightley, 1989; Ward, 1990). For example, many phenotypic values reflect the accumulation of metabolic end-products (such as the concentrations or the weights of metabolites, e.g. the degree of fatness, strength of pigmentation, etc.) and these can be regarded as some function of the integral of flux over time. Some phenotypic values may be more direct functions of flux, such as measures of metabolic rate, the degrees of responses to environmental stimuli or measures of power output. Other phenotypic values which are other functions of metabolic flux can also be envisaged.

An advantage of viewing quantitative inheritance in terms of the inheritance of metabolic flux is that phenotypic value is thereby related explicitly to the catalytic activity of gene products and these in turn represent the quantitative expression of genetic information at its most fundamental level. A biologically based, genotype-phenotype mapping function is thus defined (in principle at least) which relates phenotypic value to the genetic information carried on the chromosomes, and is expressed in terms of the biochemical kinetics of the gene products catalysing the biochemical network. 
The simplest possible model for a polygenicallydetermined quantitative trait concerns the simple linear pathway, i.e. a chain of unsaturated, reversible, monomolecular reactions at equilibrium and in the absence of feedback inhibition and other non-linearities. This pathway can be represented as:

$S_{0} \rightleftharpoons S_{1} \rightleftharpoons \ldots \rightleftharpoons S_{n}$,

in which a metabolite of concentration $S_{0}$ is converted into a metabolite of concentration $S_{n}$ via a chain of reversible, monomolecular biochemical reactions. Each step in the chain is catalysed by the product of a separate gene locus. For this simple pathway, the rate of flow $J$ through the entire pathway is given by:

$J=\frac{\left(S_{0}-S_{n}\right) / K_{0, n}}{\sum_{i=1}^{n} K_{0, i-1} / a_{i}}$

(Kacser \& Burns, 1973, 1981; Heinrich \& Rapoport, 1974). Here $J$ represents metabolic flux, $a_{i}$ is the genetically-determined catalytic activity of the product of the $i$ th gene locus (measured as the ratio of the maximal reaction velocity to the Michaelis constant relative to the enzyme substrate), $K_{i, j}$ is the chemical equilibrium constant from the $i$ th step up to and including the $j$ th step in the pathway (with $K_{0,0}=1$ ), $n$ is the number of gene loci whose products catalyse the pathway and $S_{i}$ is the concentration of the $i$ th metabolite.

On the basis of eqn 1, a genotype-phenotype mapping function for metabolic flux through a simple linear pathway can be proposed as:

$J=\frac{E}{G}$,

where $G=\sum_{i=1}^{n}\left(x_{i}+y_{i}\right)$

(Ward, 1990). Here, $x_{i}$ and $y_{i}$ are, respectively, maternally- and paternally-derived 'allelic values' at the $i$ th gene locus, representing maternal and paternal contributions to the catalytic activity of the product of the $i$ th locus, and $E$ is an 'all the rest' component comprising contributions to $J$ from factors external to the pathway itself, including environmental and background genetic effects (see Ward (1990) for further details). The additively-determined genotypic value $G$ has also been called a 'group enzyme activity' by Keightley \& Kacser (1987) and Keightley (1989).

Eqn 2 is based on many restrictive assumptions but is useful as a means of illustrating important principles which hold for more general models (of more complex pathways). Firstly, most biochemical networks contain linear segments whose properties approximate those of the simple linear pathway. Eqn 2 shows that the flux through these linear segments is a function of an additively-determined genotypic value $G$. The flux through a more complex pathway will also be a function of such additively-determined genotypic values, one for each linear section of the pathway. As an illustration, the slightly more complex pathway illustrated in Fig. 1 consists of three simple linear pathways sharing an intermediary metabolite. The genotype-phenotype mapping function for the flux through the branch on the upper right of Fig. 1 is given by:

$J=\frac{E_{1} G_{C}-E_{2} G_{A}}{G_{A} G_{B}+G_{A} G_{C}+G_{B} G_{C} / K_{A, S}}$,

where $G_{A}, G_{B}$ and $G_{C}$ are the group enzyme activities (additively determined genetic components) for the fluxes through each of the three linear pathways $A \rightleftharpoons S, \quad S \rightleftharpoons B$ and $S \rightleftharpoons C$ and where $E_{1}=S_{A}-S_{B} / K_{A, B}$ and $E_{2}=S_{C} / K_{S, B}-S_{B}-S_{B} / K_{S, B}$ (Keightley \& Kacser, 1987; Keightley, 1989).

Secondly, it can be asserted that the mathematical relationship between $J$ and any of its additive genetic components is never a simple linear function. The simplest biological model of polygenically-determined quantitative traits leads to a genotype-phenotype mapping function which is an inverse (or hyperbolic) function of an additively-determined genetic component $G$ (eqn 2). Models of more complex pathways will lead to yet more complex genotype-phenotype mapping functions than this (e.g. eqn 3). Such mapping functions will never be simple linear functions of the additive genetic components.

These ideas are now expressed more formally. A phenotypic value $P$ which is the outcome of a complex biochemical network can be viewed as a mathematical

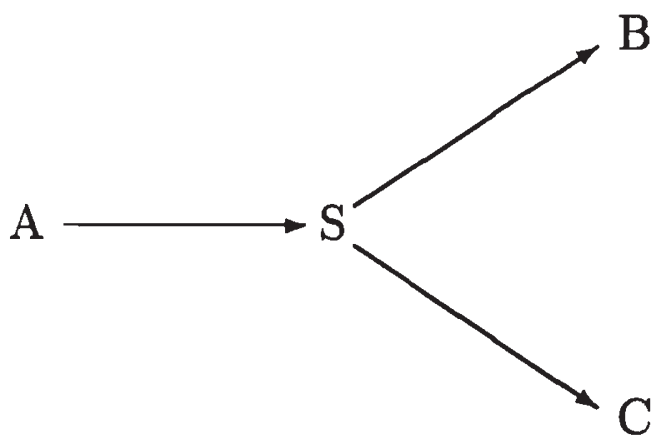

Fig. 1 Simple branched pathway. Each arrow represents a simple linear pathway (see text) and these pathways share an intermediary metabolite $S$. Metabolite $A$ is converted into metabolite $S$, which then serves as a substrate for two separate pathways, one converting $S$ into $B$ and the other converting $S$ into $C$. 
function of a vector $\mathbf{g}$ of additively-determined genotypic values, i.e. as a function of:

$\mathbf{g}=\left(\begin{array}{c}G_{1} \\ G_{2} \\ \vdots \\ G_{m}\end{array}\right)=\left(\begin{array}{c}\sum_{i=1}^{n_{1}}\left(x_{1 i}+y_{1 i}\right) \\ \sum_{i=1}^{n_{2}}\left(x_{2 i}+y_{2 i}\right) \\ \vdots \\ \sum_{i=1}^{n_{m}}\left(x_{m i}+y_{m i}\right)\end{array}\right)$

where $m$ is the number of linear segments in the biochemical network underlying the expression of the trait. The genotype-phenotype mapping function can be stated as:

$P=f(\mathbf{g}, E)+\varepsilon$,

where $E$ is an 'all the rest' component including environmental effects and background genetic effects, and $\varepsilon$ is an uncorrelated, random measurement error with zero expectation. Nothing can be said at this stage about the genotype-phenotype mapping function $f$, except that it is non-linear in $\mathbf{g}$.

It is shown below that the non-linearity itself of the genotype-phenotype mapping function is sufficient to allow parent-offspring regression to increase in strength with the extremity of the environment, in direct response to an increase in the average value of $E$. In order to make this point it will first be necessary to establish some basic results concerning the inheritance of the additive genetic component $\mathbf{g}$.

\section{Within-sibship mean and variance of the additive genetic component}

The existence of an additive genetic component $\mathbf{g}$ within eqn 5 renders feasible some mathematical analysis of the parent-to-offspring transmission of $P$ in the absence of any knowledge of the genotype-phenotype mapping function $f$.

For simplicity, consider first the parent-to-offspring transmission of a single additive genetic component (i.e. only one of the elements of the vector g). Let the value of this component within an individual be $G=\sum_{i=1}^{n}\left(x_{i}+y_{i}\right)$. An associated heterozygosity $H$ and degree of linkage coupling $C$ for that additive genetic component within that individual can also be defined as:

$$
\begin{aligned}
H & =\sum_{i=1}^{n}\left(x_{i}-y_{i}\right)^{2}, \\
C & =\sum_{i<j \leq n}\left(x_{i}-y_{i}\right)\left(x_{j}-y_{j}\right)\left(1-2 r_{i j}\right),
\end{aligned}
$$

respectively, where $r_{i j}$ is the recombination fraction between the $i$ th and $j$ th loci within the $n$-locus geno- type, with $0 \leq r_{i j} \leq 0.5$. The $C$ measures the extent to which alleles of larger effect among heterozygous loci are linked on the same chromosomal strand.

Consider now a mating of two individuals whose values for $G, H$ and $C$ are respectively $G_{1}, H_{1}, C_{1}$ and $G_{2}, H_{2}, C_{2}$. The mean and variance of $G$ within the sibship produced by this mating follow directly as:

$\mu\left(G \mid G_{1}, G_{2}\right)=\frac{1}{2}\left(G_{1}+G_{2}\right)$,

$\operatorname{Var}\left(G \mid H_{1}, C_{1}, H_{2}, C_{2}\right)=\frac{1}{4}\left(H_{1}+H_{2}\right)+\frac{1}{2}\left(C_{1}+C_{2}\right)$,

(Ward, 1990). Here, ' $\mu$ ' and 'Var' signify the mean and the variance, respectively.

Consider now the entire vector $\mathbf{g}$ of additive genetic components (eqn 4) within a single individual. Let $\mathbf{H}$ refer to a diagonal matrix of heterozygosities, one diagonal element corresponding to each element of $\mathbf{g}$, i.e.

$\mathbf{H}=\left(\begin{array}{cccc}H_{1} & 0 & \ldots & 0 \\ 0 & H_{2} & \ldots & 0 \\ \vdots & \vdots & \ddots & \vdots \\ 0 & 0 & \ldots & H_{m}\end{array}\right)$

Let $\mathbf{C}$ refer to a matrix of degrees of linkage coupling of the genes within that individual, i.e.

$\mathbf{C}=\left(\begin{array}{cccc}C_{11} & C_{12} & \ldots & C_{1 m} \\ C_{21} & C_{22} & \ldots & C_{2 m} \\ \vdots & \vdots & \ddots & \vdots \\ C_{m 1} & C_{m 2} & \ldots & C_{m m}\end{array}\right)$.

Within this latter matrix, each diagonal element $C_{i i}$ is the degree of linkage coupling among the heterozygous loci of the $i$ th additive genetic component of $\mathbf{g}$, whereas an off-diagonal element $C_{i j}$ is the degree of linkage coupling of the heterozygous loci of the $i$ th additive genetic component to the heterozygous loci of the $j$ th additive genetic component.

From a mating of two individuals whose values are $\mathbf{g}_{1}, \mathbf{H}_{1}, \mathbf{C}_{1}$ and $\mathbf{g}_{2}, \mathbf{H}_{2}, \mathbf{C}_{2}$, respectively, the mean and variance of $\mathbf{g}$ within the resultant sibship are given by:

$\mu\left(\mathbf{g} \mid \mathbf{g}_{1}, \mathbf{g}_{2}\right)=\frac{1}{2}\left(\mathbf{g}_{1}+\mathbf{g}_{2}\right)$

$\operatorname{Var}\left(\mathbf{g} \mid \mathbf{H}_{1}, \mathbf{C}_{1}, \mathbf{H}_{2}, \mathbf{C}_{2}\right)=\frac{1}{4}\left(\mathbf{H}_{1}+\mathbf{H}_{2}\right)+\frac{1}{2}\left(\mathbf{C}_{1}+\mathbf{C}_{2}\right)$. 
These results are exact and make no assumptions about the number of additive genetic components, the number of gene loci contributing to each additive genetic component, the allelic values at each gene locus and the recombination fractions among the gene loci.

Another important result is that any additive genetic component $G$ within an individual is uncorrelated with its associated $H$ and $C$ within the individual. This is demonstrated in the Appendix. It follows from this that, within an individual, any element $G_{i}$ of $\mathbf{g}$ is uncorrelated with any heterozygosity $H_{j}$ and/or degree of linkage coupling $C_{k}$ associated, respectively, with any other elements $G_{j}$ and $G_{k}$ of $\mathbf{g}$ within that individual.

A great deal of information is therefore available concerning the parent-to-offspring transmission of an additive genetic component. This information is used below to obtain an approximation to the parent-offspring regression coefficient. This approximation is expressed in terms of the genetic and environmental parameters of the genotype-phenotype mapping function defined by eqn 5 .

\section{Parent-offspring regression coefficient}

Although the mathematical function $f$ in eqn 5 is unknown, approximate expressions for the central moments of $P$ can be written down using truncated Taylor series expansions. Consider a mating between two randomly chosen individuals whose phenotypic values are $P_{1}=f\left(\mathbf{g}_{1}, E_{1}\right)+\varepsilon_{1}$ and $P_{2}=f\left(\mathbf{g}_{2}, E_{2}\right)+\varepsilon_{2}$, respectively. The mean phenotypic value of the resultant sibship is approximately:

$$
\begin{aligned}
\mu\left(P \mid P_{1}, P_{2}\right) & \approx f\left[\frac{1}{2}\left(\mathbf{g}_{1}+\mathbf{g}_{2}\right), \mu_{w}(E)\right] \\
& +\phi_{1}^{T}\left[\frac{1}{4}\left(\mathbf{H}_{1}+\mathbf{H}_{2}\right)+\frac{1}{2}\left(\mathbf{C}_{\mathbf{1}}+\mathbf{C}_{2}\right)\right] \phi_{1} f,
\end{aligned}
$$

where $\phi_{1}=\frac{\partial}{\partial \mathbf{g}}$ operating on $f$,

and where $\mu_{w}(E)$ represents the within-sibship mean value of $E$. Eqn 6 is a second-order (quadratic) approximation to the within-sibship mean. It ignores contributions to the mean and variance of $P$ from central moments of $\mathbf{g}$ and $E$ of order higher than two. All vectors are column vectors and the superscript ' $T$ ' denotes vector/matrix transposition. The partial derivatives defined by the vector operator $\phi_{1}$ are evaluated at the within-sibship mean values of $\mathbf{g}$ and $E$.
Recall that for any plot of $y$ against $x$, the slope of the least-squares line is $\operatorname{Cov}(x, y) / \operatorname{Var}(x)$, where 'Cov' denotes covariance. Under the general model defined by eqn 5 , the parent-offspring regression coefficient $\beta_{O \tilde{P}}$ is therefore:

$\beta_{O P}=\frac{\operatorname{Cov}\left[\frac{1}{2}\left[f\left(\mathbf{g}_{1}, E_{1}\right)+\varepsilon_{1}+f\left(\mathbf{g}_{2}, E_{2}\right)+\varepsilon_{2}\right], \mu\left(P \mid P_{1}, P_{2}\right)\right]}{\operatorname{Var}\left[\frac{1}{2}\left(P_{1}+P_{2}\right)\right]}$.

Under random mating, the denominator of $(7)$ is simply $(1 / 2) \operatorname{Var}(P)$. By applying approximation 6 for $\mu\left(P \mid P_{1}\right.$, $P_{2}$ ), expanding the numerator of (7) as a Taylor series (refer to eqn 10 in the Appendix) and ignoring contributions from central moments of order higher than two, one obtains the following quadratic approximation for $\beta_{O P}$ :

$\beta_{O P} \approx \frac{\phi_{2}^{I} \operatorname{Var}(\mathbf{g}) \phi_{2}-\{\mu(P)-f[\mu(\mathbf{g}), \mu(E)]\}^{2}}{\operatorname{Var}(P)}$,

where $\phi_{2}=\frac{\partial P}{\partial \mathbf{g}}$.

Here, $\mu(P), \mu(\mathbf{g})$ and $\mu(E)$ refer to the population mean values of $P, \mathbf{g}$ and $E$, respectively and $\operatorname{Var}(P)$ $\operatorname{Var}(\mathbf{g})$ and $\operatorname{Var}(E)$ refer to their population variances. The relative simplicity of eqn 8 stems from the lack of covariance between $\mathbf{g}$ and either $\mathbf{H}$ or $\mathbf{C}$ (see Appendix): and hence the contribution to $\beta_{O \dot{P}}$ from the second part on the right hand side of eqn 6 is zero.

The partial derivatives in eqn 8 , defined by $\phi_{2}$, are evaluated at $\mu(\mathbf{g})$ and $\mu(E)$. They express the slope of the genotype-phenotype mapping function at $\mu(\mathbf{g})$ and $\mu(E)$ and their values are not constant in response to a change of either $\mu(\mathbf{g})$ or $\mu(E)$ due to the non-linearity of the genotype-phenotype mapping function. The $\phi_{2}$ can therefore be regarded as a function of $\mu(\mathbf{g})$ and $\mu(E)$. Its appearance in eqn 8 indicates that $\beta_{O \bar{P}}$ is a function of $\mu(E)$ and that this effect stems from the non-linearity of the genotype-phenotype mapping function.

In order to illustrate this point, the genotype-phenotype mapping function for the simple linear metabolic pathway is $P=E / G$ (eqn 2) and the parent-offspring regression coefficient follows as:

$\beta_{O \dot{P}} \approx \frac{\operatorname{Var}(G) \frac{\mu^{2}(E)}{\mu^{4}(G)}-\left[\mu(P)-\frac{\mu(E)}{\mu(G)}\right]^{2}}{\operatorname{Var}(P)}$ 
This equation illustrates explicitly that the parentoffspring regression coefficient for metabolic flux through a simple linear pathway is a direct function of $\mu(E)$.

\section{Discussion}

The above analysis shows that, where the genotypephenotype mapping function is non-linear in the additive genetic component of the phenotype, the parent-offspring regression coefficient $\beta_{O \dot{P}}$ is a function of the population means (as well as the variances) of the genetic and environmental parameters. In eqn 8, this dependence on means occurs via $\phi_{2}$ which is a function of the population mean values of $E$ and $\mathrm{g}$. It is also pointed out here that if phenotypic value is the outcome of metabolic flux through a complex biochemical network, then the genotype-phenotype mapping function is always a non-linear function of an additive genetic component $\mathrm{g}$ defined by eqn 4 . Here, 'complex' means any pathway of more than one biochemical reaction. Most phenotypic values would fulfil this requirement.

The numerous empirical observations of an increase in the strength of parent-offspring regression coefficient (narrow heritability) with the extremity of the environment could therefore reflect increases of $\mu(E)$, i.e. increases in the average contribution of factors outside the biochemical network itself. From a biological perspective, it is entirely reasonable to propose that this component might increase with the extremity of the environmental conditions. More generally, the present analysis shows that empirical observations of elevated $\beta_{O \bar{P}}$ under stressful environments do not necessarily indicate that any change has taken place in either the mean or the variance of the additive genetic component $\mathbf{g}$ of phenotypic value following the transfer of the study population to the extreme environment.

Eqn 6 ignores any contribution to $\beta_{O \bar{P}}$ from common within-family environmental factors, i.e. it ignores the possibility that the environmental contributions to the offspring phenotypes are correlated with the environmental contributions to the phenotypes of their parents. The effect of such factors would be the addition of another term $\phi_{3} \operatorname{Var}\left(E_{c}\right)$ to the numerator of eqn 6 , where $E_{c}$ represents such a common withinfamily environmental factor, and where $\phi_{3}=\partial P / \partial E$ evaluated at the population mean values of $\mathbf{g}$ and $E$. Incorporating such effects into the analysis would not change the conclusions of this paper.

There are some important differences between the model of quantitative inheritance developed here and that of the classical additive-dominance model (Mather \& Jinks, 1971; Bulmer, 1985; Falconer, 1989). Under the approach taken here, the genetic and environ- mental parameters of an individual's phenotypic value are properties of the constitution of that individual itself rather than of the population. One advantage in taking this approach is that the explanation for increased heritability with the extremity of the environment is intrinsic to the resultant mathematical model. In contrast, under the classical quantitative genetics formulation, the parameters of an individual's phenotypic value are not properties of that individual itself but are population parameters defined entirely in statistical terms. Furthermore, these parameters are defined at the phenotypic level and do not reflect any biological model of gene expression. Under this classical approach, the parent-offspring regression coefficient is a function only of the variances of genetic and environmental parameters: it contains no contributions from the mean values of the genetic and environmental parameters of the phenotype. Hence, an explanation for the data cited concerning extreme environments is not intrinsic to the mathematical model against which the data are interpreted. This has obliged researchers to formulate an explanation a posteriori for these data in a way extraneous to the additive-dominance model.

The important mathematical difference between the present and the classical approaches is that the classical additive-dominance model is based on the supposition that the genotype-phenotype mapping function is linear, e.g. $P=G+E$. Under such a linear model, the partial derivatives represented by $\phi_{2}$ in eqn 8 would each be equal to one regardless of the population mean values of $\mathbf{g}$ and $E$. The parent-offspring regression coefficient would then be a function only of the variances of the genetic and environmental parameters of the linear model. This supposition of a linear genotype-phenotype mapping function is justified by the operational definitions given for the parameters of that model (Falconer, 1989). The resultant methodology has proved of invaluable use as a guide to selective breeding. However, the parameters of the classical model do not reflect any model of development or gene expression and they have meaning only within the environment and population for which they have been estimated. The analysis presented here shows that care must be taken when interpreting changes in the values of the parameters of the additive-dominance model following changes in the experimental conditions. The classical methodology gives no way of inferring the biological meaning of such changes in parameter values.

\section{References}

BARTON, N. H. AND TURELLI, M. 1989. Evolutionary quantitative genetics: how little do we know? Ann. Rev. Genet., 23, $337-370$. 
BEAUMONT, M. A. 1988. Stabilizing selection and metabolism. Heredity, 61, 433-438.

BELYAEV, D. K. AND BORODIN, P. M. 1982. The influence of stress on variation and its role in evolution. Biol. Zbl., 100 , $705-714$.

BULMER, M. G. 1985. The Mathematical Theory of Quantitative Genetics. Clarendon Press, Oxford.

CROW, J. F. 1957. Genetics of insecticide resistance to chemicals. Ann. Rev. Entomol., 2, 227-236.

DERR, J. A. 1980. The nature of variation in life history characters of Dysdercus bimaculatus (Heteroptera: Pyrrhocoridae), a colonising species. Evolution, 34, 548-557.

EBERT, D., YAMPOLSKY, L. AND STEARNS, S. C. 1993. Genetics of life history in Daphnia magna. I. Heritabilities at two food levels. Heredity, 70, 335-343.

FALCONER, D. S. 1989. Introduction to Quantitative Genetics, 3rd edn. Longman, London.

FISHER, R. A. 1918. The correlation between relatives on the supposition of mendelian inheritance. Trans. $R$. Soc. Edinb., 52, 399-433.

HEINRICH, R. AND RAPOPORT, T. A, 1974. A linear steady-state treatment of enzymatic chains: general properties, control and effector strength. Eur. J. Biochem., 42, 89-95.

HOFFMAN, A. A. AND PARSONS, P. A. 1991. Evolutionary Genetics and Environmental Stress. Oxford University Press, New York.

HOLLOWAY, G. J., POVEY, S. R. AND SIBLY, R. M. 1990. The effect of new environment on adapted genetic architecture. Heredity, 64, 323-330.

KACSER, H. ANd burns, J. A. 1973. The control of flux. Symp. Soc. Exp. Biol., 27, 65-104.

KACSER, H. AND BURNS, J. A. 1981. The molecular basis of dominance. Genetics, 97, 639-666.

KEIGHTLEY, P. D. 1989. Models of quantitative variation of flux in metabolic pathways. Genetics, 121, 869-876.

KEIGHTLEY, P. D. AND KACSER, H. 1987. Dominance, pleiotropy and metabolic structure. Genetics, 117, 319-329.

LAGRIDGE, J. AND GRIFFING, B. 1959. A study of high temperature lesions in Arabidopis thaliana. Aust. J. Biol. Sci., 12, $117-135$.

MARTIN, N. G., OAKSHOTT, J. G., GIBSON, J. B., STARMER, G. A., PERL, J. AND wILKS, A. v. 1985. A twin study of psychomotor and physiological responses to an acute dose of alcohol. Behav. Genet., 15, 305-347.

MATHER, K. AND JINKS, J. L, 1971. Biometrical Genetics, 2nd edn. Chapman \& Hall, London.

PAN1, S. N. AND LASLEY, J. F. 1972. Genotype $\times$ Environment Interactions in Animals: Theoretical Considerations and Review Findings. Research Bulletin no. 992. Agricultural Experiment Station, University of Missouri-Columbia, Columbia, MO.

PARSONS, P. A. 1982. Acetic acid vapour as a resource and stress in Drosophila. Aust. J. Zool., 30, 427-433.

SCHNEE, F. B. AND THOMPSON, J. N. Jr. 1984. Conditional polygenic effects in the stearnopleural bristle system of Drosophila melanogaster. Genetics, 108, 409-424.

URQuharT, c. 1971. Genetics of lead tolerance in Festuca ovina. Heredity, 26, 19-33.
VAN HERREWEGE, J. AND DAVID, J. R. 1984. Extension of life duration by dietary ethanol in Drosophila melanogaster: response to selection in two strains of different origins. Genetica, 63, 61-70.

WARd, P. J. 1985. An Analysis of the Species' Border Problem. Ph.D. Thesis, La Trobe University, Bundoora, Australia.

WARD, P. J. 1990. The inheritance of metabolic flux: expressions for the within-sibship mean and variance given the parental genotypes. Genetics, 125, 655-667.

WESTERMAN, J. M. AND PARSONS, P. A. 1973. Variation in genetic architecture at different doses of $\gamma$-radiation as measured by longevity in Drosophila melanogaster. Can. J. Genet. Cytol., 15, 289-298.

\section{Appendix}

It is shown here that the covariance of $G$ with $C$ (see text) is zero. This covariance can be written down as:

$$
\begin{gathered}
\operatorname{Cov}\left[\sum_{i=1}^{n} x_{i}+y_{i}, \sum_{i<k \leq n}\left(x_{j}-y_{j}\right)\left(x_{k}-y_{k}\right)\left(1-2 r_{j k}\right)\right] \\
=\sum_{i=1}^{n} \sum_{j<k \leq n}\left(1-2 r_{j k}\right) \operatorname{Cov}\left[x_{i}+y_{i},\left(x_{j}-y_{j}\right)\left(x_{k}-y_{k}\right)\right] .
\end{gathered}
$$

By showing that this covariance is zero, the covariance of $G$ with $H$ is simultaneously shown to be zero as this is simply a special case $k=j$ and $r_{j k}=0$ of the general proof presented here.

The covariance term $\operatorname{Cov}\left[x_{i}+y_{i},\left(x_{j}-y_{j}\right)\left(x_{k}-y_{k}\right)\right]$ can be solved via a general equation for the covariance of functions of two sets of random variables. The covariance of any smooth function $h_{1}\left(s_{1}, s_{2}, \ldots\right)$ of a set of random variables $s_{1}, s_{2}, \ldots$, with another smooth function $h_{2}\left(t_{1}, t_{2}, \ldots\right)$ of a second set of random variables $t_{1}, t_{2}, \ldots$, is given by:

$$
\begin{aligned}
\operatorname{Cov} & {\left[h_{1}\left(s_{1}, s_{2}, \ldots\right), h_{2}\left(t_{1}, t_{2}, \ldots\right)\right] } \\
& =\sum_{i} \sum_{j} \operatorname{Cov}\left(s_{i}, t_{j}\right) \frac{\partial h_{1}}{\partial s_{i}} \frac{\partial h_{2}}{\partial t_{j}} \\
& -\left[\frac{1}{2} \sum_{i} \operatorname{Var}\left(s_{i}\right) \frac{\partial^{2} h_{1}}{\partial s_{i}^{2}}+\sum_{i<j} \operatorname{Cov}\left(s_{i}, s_{j}\right) \frac{\partial^{2} h_{1}}{\partial s_{i} \partial s_{j}}\right] \\
& \times\left[\frac{1}{2} \sum_{i} \operatorname{Var}\left(t_{i}\right) \frac{\partial^{2} h_{2}}{\partial t_{i}^{2}}+\sum_{i<j} \operatorname{Cov}\left(t_{i}, t_{j}\right) \frac{\partial^{2} h_{2}}{\partial t_{i} \partial t_{j}}\right]+\ldots,
\end{aligned}
$$

where '..' means other terms involving third and higher-order derivatives. The partial derivatives are all evaluated at the mean values of $s_{1}, s_{2}, \ldots$ and $t_{1}, t_{2}, \ldots$. 
For the present problem, $h_{1}\left(x_{i}, y_{i}\right)=x_{i}+y_{i}$ and $h_{2}\left(x_{j}, y_{j}, x_{k}, y_{k}\right)=\left(x_{j}-y_{j}\right)\left(x_{k}-y_{k}\right)$. In this case, the terms involving higher-order derivatives are all zero. The covariance of $\left(x_{i}+y_{i}\right)$ and $\left(x_{j}-y_{j}\right)\left(x_{k}-y_{k}\right)$ then comes down to:

$$
\begin{aligned}
& \operatorname{Cov}\left[x_{i}+y_{i},\left(x_{j}-y_{j}\right)\left(x_{k}-y_{k}\right)\right] \\
& =\left[\mu\left(x_{k}\right)-\mu\left(y_{k}\right)\right] \operatorname{Cov}\left(x_{i}, x_{j}\right)-\left[\mu\left(x_{k}\right)-\mu\left(y_{k}\right)\right] \operatorname{Cov}\left(x_{i}, y_{j}\right) \\
& +\left[\mu\left(x_{j}\right)-\mu\left(y_{j}\right)\right] \operatorname{Cov}\left(x_{i}, x_{k}\right)-\left[\mu\left(x_{j}\right)-\mu\left(y_{j}\right)\right] \operatorname{Cov}\left(x_{i}, y_{k}\right) \\
& +\left[\mu\left(x_{k}\right)-\mu\left(y_{k}\right)\right] \operatorname{Cov}\left(y_{i}, x_{j}\right)-\left[\mu\left(x_{k}\right)-\mu\left(y_{k}\right)\right] \operatorname{Cov}\left(y_{i}, y_{j}\right) \\
& +\left[\mu\left(x_{j}\right)-\mu\left(y_{j}\right)\right] \operatorname{Cov}\left(y_{i}, x_{k}\right)-\left[\mu\left(x_{j}\right)-\mu\left(y_{j}\right)\right] \operatorname{Cov}\left(y_{i}, y_{k}\right) .
\end{aligned}
$$

The solution of eqn 8 in the text assumes that the parental pairs are sampled at random from the general population. Under this assumption, the maternallyand paternally-derived alleles (the $x \mathrm{~s}$ and the $y \mathrm{~s}$ ) at any gene locus within any parental pair are sampled from a common distribution. All of the terms $\mu(x)-\mu(y)$ are therefore zero in the above expression. Substituting this result back into eqn 9 shows that the covariance of $G$ and $C$ is zero. 